\title{
Deterministic Electrical Charge-State Initialization of Single Nitrogen-Vacancy Center in Diamond
}

\author{
Y. Doi, ${ }^{1}$ T. Makino, ${ }^{2,3}$ H. Kato, ${ }^{2,3}$ D. Takeuchi, ${ }^{2,3}$ M. Ogura, ${ }^{2,3}$ H. Okushi, ${ }^{2,3}$ H. Morishita, ${ }^{1,3}$ T. Tashima, ${ }^{1}$ S. Miwa, ${ }^{1}$ \\ S. Yamasaki, ${ }^{2,3}$ P. Neumann, ${ }^{4}$ J. Wrachtrup, ${ }^{4}$ Y. Suzuki, ${ }^{1,3}$ and N. Mizuochi ${ }^{1,2,3, *}$ \\ ${ }^{1}$ Graduate School of Engineering Science, Osaka University, Toyonaka, Osaka 560-8531, Japan \\ ${ }^{2}$ Energy Technology Research Institute-National Institute of Advanced Industrial Science and Technology, \\ Tsukuba, Ibaraki 305-8568, Japan \\ ${ }^{3}$ CREST, Japan Science and Technology Agency, Kawaguchi, Saitama 332-0012, Japan \\ ${ }^{4}$ 3. Physikalisches Institut, Universität Stuttgart, Pfaffenwaldring 57, D-70550 Stuttgart, Germany
}

(Received 30 October 2013; published 31 March 2014)

\begin{abstract}
Apart from applications in classical information-processing devices, the electrical control of atomic defects in solids at room temperature will have a tremendous impact on quantum devices that are based on such defects. In this study, we demonstrate the electrical manipulation of individual prominent representatives of such atomic solid-state defects, namely, the negative charge state of single nitrogenvacancy defect centers $\left(\mathrm{NV}^{-}\right)$in diamond. We experimentally demonstrate, deterministic, purely electrical charge-state initialization of individual NV centers. The NV centers are placed in the intrinsic region of a $p-i$ - $n$ diode structure that facilitates the delivery of charge carriers to the defect for charge-state switching. The charge-state dynamics of a single NV center were investigated by time-resolved measurements and a nondestructive single-shot readout of the charge state. Fast charge-state switching rates (from negative to neutrally charged defects), which are greater than $0.72 \pm 0.10 \mu \mathrm{s}^{-1}$, were realized. Furthermore, in no-operation mode, the realized charge states were stable for presumably much more than $0.45 \mathrm{~s}$. We believe that the results obtained are useful not only for ultrafast electrical control of qubits, long $T_{2}$ quantum memory, and quantum sensors associated with single NV centers but also for classical memory devices based on single atomic storage bits working under ambient conditions.
\end{abstract}

DOI: 10.1103/PhysRevX.4.011057

\section{INTRODUCTION}

State-of-the-art devices have sizes of a few tens of nanometers, owing to electrical control, which is superior to optical control, in which diffraction sets limits that are 1 order of magnitude larger. The electrical control of single atomic units in, e.g., single $\mathrm{P}$ dopants in silicon (Si:P) [1,2] and quantum-dots in GaAs [3,4] is getting common practice nowadays. Owing to their single quantum nature, quantum systems have led to the development of novel fields such as quantum electronics, spintronics, and metrology. However, a severe limitation for such quantum systems is the requirement of cryogenic conditions for operation, which can be overcome by using other host materials. Here, we demonstrate the deterministic and purely electrical charge-state control of single atomic defects in diamond at room temperature. For this purpose, we use single nitrogen-vacancy (NV)

\footnotetext{
* Corresponding author. mizuochi@mp.es.osaka-u.ac.jp

Published by the American Physical Society under the terms of the Creative Commons Attribution 3.0 License. Further distribution of this work must maintain attribution to the author(s) and the published article's title, journal citation, and DOI.
}

Subject Areas: Nanophysics, Semiconductor Physics

centers that primarily appear in two charge states (i.e., negatively or neutrally charged, $\mathrm{NV}^{-}$or $\mathrm{NV}^{0}$ ).

The negatively charged NV center in diamond [Fig. 1(a)] is a promising candidate for a solid-state room-temperature quantum bit and quantum sensor with various applications in quantum information [5-9], magnetometry [10-13], and biosensing [14-16]. The electron and proximal nuclear spins [17] of individual $\mathrm{NV}^{-}$centers can be manipulated and read optically [18]. Recently, it was reported that stochastic transitions between $\mathrm{NV}^{-}$and $\mathrm{NV}^{0}$ are induced by laser illumination [19-22], surface termination [23-25], and combined optical and electrical operation [7,26,27]. Very recently, purely electrical operations yielded the first room-temperature single photon source $[7,28]$. However, to date, deterministic charge-state control was only possible via particular optical means on submillisecond time scales [22,29], which is too slow for many interesting future applications.

The fast and deterministic charge-state control of single NV centers is essential for quantum applications. For example, charge-state stabilization improves qubit initialization and readout. In addition, fast and deterministic changes of the charge state enable fast switching of hyperfine interactions among spin qubits, which is vital for preserving spin quantum states. Furthermore, stochastic charge-state changes lead to spectral diffusion of the $\mathrm{NV}^{-}$ 
(a)

(c)
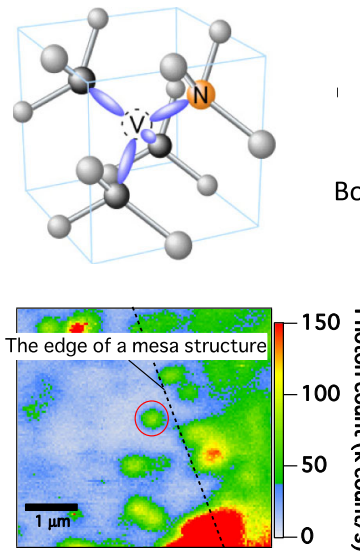

(b)

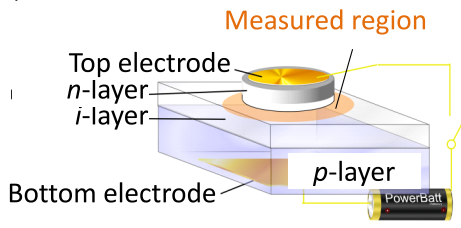

(d)

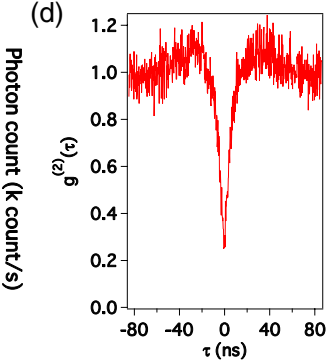

FIG. 1. (a) Structure of a NV center comprising a substitutional nitrogen atom and a neighboring vacancy in the diamond lattice. (b) Sketch of the $p-i-n$ structure of the diamond diode. The thicknesses of the $p$-type, intrinsic, and $n$-type layers of the device are $0.5 \mathrm{~mm}, 10 \mu \mathrm{m}$, and $500 \mathrm{~nm}$, respectively. (c) Confocal microscopy PL raster scan image of the intrinsic layer. All measurements (except the distance-dependence measurement) were conducted on the NV center circled in red. (d) The corresponding antibunching statistics of the NV center in (c). $\mathrm{g}^{(2)}(0)<0.5$ indicates photons originating from a single center.

zero-phonon line (ZPL) [30-32], which is detrimental to the efficiency of two-photon quantum interference [5] and related quantum communication applications. In contrast, stochastic, optically induced charge-state switching using high laser power (about $5 \mathrm{MW} / \mathrm{cm}^{2}$ ) can be exploited for the decoupling of nuclear spins from the NV electron spin, yielding nuclear spin coherence times $\left(T_{2}\right)$ in diamond at room temperature [6]. Potentially, the $T_{2}$ of the nuclear spin is expected to be longer than $1 \mathrm{~h} \mathrm{[6]}$; however, the highpower laser illumination heats the diamond surface and causes drifting in the electron spin resonance transition [33], which reduces $T_{2}$. In contrast, the $T_{2}$ of the nuclear spin may be made longer by electrical control, owing to its potential for fast and efficient control. The present optical charge-state switching mechanism $\left(\mathrm{NV}^{-}\right.$to $\left.\mathrm{NV}^{0}\right)$ involves orange laser light (from about $\sim 580$ to $\sim 640 \mathrm{~nm}$ ) pumping and is rather slow (> kHz [22]) because two-photon absorption processes are involved [22,29]. Furthermore, the optical control efficiency is not $100 \%$ [22,29] because of non-negligible optically induced rates in the opposite direction $\left(\mathrm{NV}^{0}\right.$ to $\left.\mathrm{NV}^{-}\right)$. In contrast, the electrical change in the charge state occurs in a single step such as capture or release of a carrier, which is important for control efficiency, suppression of heating, and fast operation speed (tens of $\mathrm{MHz} \sim \mathrm{GHz}$ ) [34]. Furthermore, the electrical control of carriers and charge of qubits is important for scalable and integrated devices, which is well investigated in quantum-dot systems $[3,4]$.

Recently, progress on the combined optical and electrical manipulation of charge states between $\mathrm{NV}^{0}$ and possibly $\mathrm{NV}^{+}$of a single $\mathrm{NV}$ center [26] and between $\mathrm{NV}^{-}$and $\mathrm{NV}^{0}$ of a NV ensemble was reported [26,27]. Grotz et al. [26] reported the use of a diamond solution-gated field-effect transistor with a hydrogen-terminated $p$-type surface as the electrical part of the manipulation of single NV centers. Simultaneously, a 532-nm-laser irradiation was applied $[26,27]$, which causes fluorescence and prevents chargestate identification. However, because of recent findings, it is now obvious that the laser application actually induces severe stochastic and ongoing charge-state switching between $\mathrm{NV}^{-}$and $\mathrm{NV}^{0}$ as well as other potential proximal defects $[22,29]$. Furthermore, this switching can lead to $\mathrm{NV}^{0}$ fluorescence when $\mathrm{NV}^{-}$is the stable charge state in the dark [29] or vice versa [21]. Consequently, extrapolation about the action of purely electrical control is hardly possible because the displayed fluorescence spectra and concluded charge states are properties of a dynamic steady state under illumination and electrical control. The invoked dynamics of electrical control in the dark (i.e., without simultaneous illumination) have not been shown or investigated yet. However, the charge state in the dark is of utmost importance to NV center applications, as they all rely on coherent spin manipulations in the dark. However, previously reported changes of fluorescence responses on the application of electrical control and laser irradiation might be a precursor of the possible effect of electrical NV control in the dark. Previously, we observed similar changes in photoluminescence (PL) spectra by simultaneously injecting current in a diamond $p-i-n$ diode [7]; thus, we further investigate the effect of current injection on NV centers. We first analyze the dynamics of the dynamic steady state by time-resolved measurements of the fluorescence response to abrupt changes in the electrical stimulus. Furthermore, we investigate the purely electrical influence on the NV center charge state, and we apply a recently developed nondestructive single-shot charge-state readout technique [35]. Consequently, we are able to perform and prove the deterministic charge-state manipulation of single NV centers in the dark.

\section{RESULTS}

\section{A. $p$ - $i-n$ diode structures in diamond containing single $\mathrm{NV}$ centers}

Efficient current injection into a single NV center requires $p$ - and $n$-type diamonds. We generated them by doping with large amounts of boron and phosphorous to induce semiconducting properties. However, those dopants generate defects that have PL in the same spectral range of the NV center. Therefore, we introduced an extremely highquality undoped $(i$-) region to form a $p-i-n$ diamond diode with mesa structure to detect single NV centers, as shown in Fig. 1(b). The synthesis and fabrication process of the $p-i-n$ diamond diode is shown in the Appendix. 
Figure 1(c) shows confocal microscopy PL raster scans of the intrinsic area. The dashed black line indicates the edge of the mesa structure. PL spectra were obtained from the single $\mathrm{NV}$ center (red circle), which is close to the edge of the mesa structure. The result of the fluorescence autocorrelation measurement of the NV center is shown in Fig. 1(d). The second-order autocorrelation function $\mathrm{g}^{(2)}(\tau)$ reveals an antibunching dip of $<0.5$ at $\tau=0$, indicating that the NV center in Fig. 1(c) is indeed a single one.

\section{B. Transient behavior of combined electrical and optical NV charge-state manipulation}

The NV center shows PL spectrum characteristics for $\mathrm{NV}^{-}$(ZPL at $637 \mathrm{~nm}$ ) without current injection, as shown in Fig. 2(a). However, the spectrum changes to $\mathrm{NV}^{0}$ (ZPL at $575 \mathrm{~nm}$ ) by simultaneous current injection, as indicated by the blue line in Fig. 2(a). The scenario in our device is as follows. Holes are injected as majority carriers from the $p$-type layer to the single $\mathrm{NV}^{-}$centers in the $i$-layer, owing to the large difference of electrons $\left(10^{13} \mathrm{~cm}^{-3}\right)$ and holes $\left(10^{16} \mathrm{~cm}^{-3}\right)$. The $\mathrm{NV}^{-}$center captures a hole and is converted to $\mathrm{NV}^{0}$ [7], which might be one process among many in the dynamic steady state.

As shown in Fig. 2(b), when the injection current is rapidly switched between 0 and $0.2 \mathrm{~mA}$ and a $675-\mathrm{nm}$ longpass filter removes the $\mathrm{NV}^{0}$ fluorescence, repetitions of decrease and recovery of the $\mathrm{NV}^{-}$fluorescence are recorded. Our results are the first real-time observation of an electrically controlled change of the dynamic steady state of a single $\mathrm{NV}^{-}$center. Note that the value of the current is sufficiently small to prevent apparent electroluminescence from the NV center, which is less than $1 \mathrm{k}$ count/s [7].

To understand the dynamics of the changes in the dynamic steady state, we performed repetitive photon accumulation measurements with higher time resolution. The pulse sequence is shown in Fig. 2(c), where we simultaneously applied a continuous-wave laser with a current pulse of $40 \mu \mathrm{s}$. The photon count decreases because of the average charge-state change induced by the current,
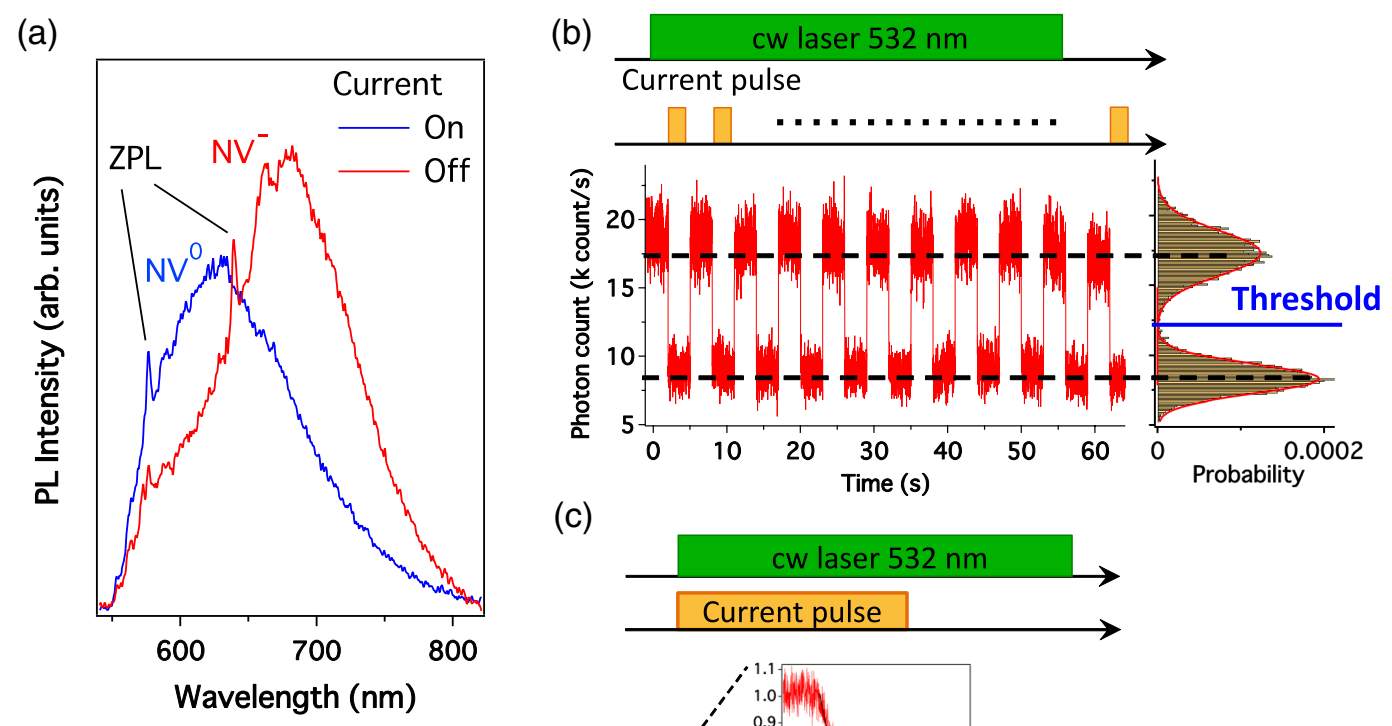

(c)

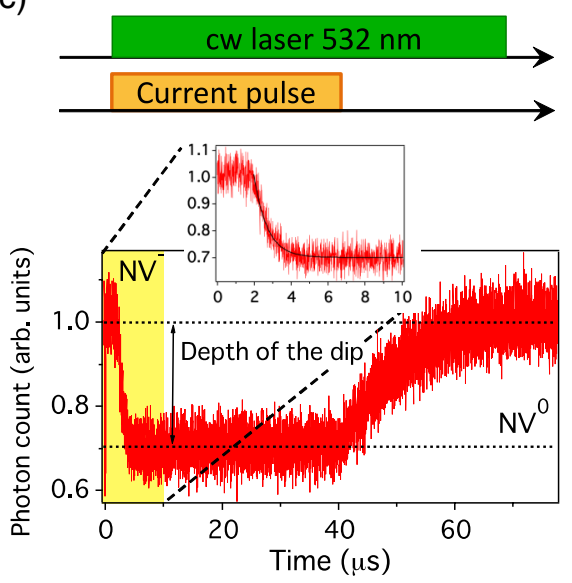

FIG. 2. (a) PL comparison of the different dynamical steady states and charge states. $\mathrm{NV}^{0}$ states were induced by current injection of $70 \mu \mathrm{A}$. The excitation laser power was $0.3 \mathrm{~mW}$. (b) Real-time trace of the fluorescence intensity with current switching between 0 and $0.2 \mathrm{~mA}$. On the upper side, the 532-nm irradiation laser light and timing of the injected current pulse are shown. The fluorescence intensity histogram is shown on the right. The solid red line was obtained from fitting of the two Gaussian distributions. The blue straight line indicates the threshold of the two dynamical steady states and charge states. (c) Charge-state transitions between $\mathrm{NV}^{-}$and $\mathrm{NV}^{0}$ in the fast time-resolution measurement, which was averaged after $4 \times 10^{6}$ repetitions. On the upper side, the 532-nm irradiation laser light $(0.2 \mathrm{~mW})$ and timing of the injected current pulse $(0.3 \mathrm{~mA})$ is shown. In the inset, the expanded region for the change from higher to lower photon count is shown. It can be fitted with a single exponential $e^{-\lambda t}$, as shown by the black line. 
similar to the results shown in Fig. 2(b). However, after the current pulse was switched off, the recovery in the photon count was slower than the initial decrease.

For understanding the charge-state dynamics, it is necessary to estimate the underlying charge-state rates. The total rate of the charge-state transition $\lambda$ comprises bidirectional rates $\lambda=\lambda^{-0}+\lambda^{0-}=\lambda_{\text {laser }}^{-0}+\lambda_{\text {current }}^{-0}+\lambda_{\text {laser }}^{0-}+$ $\lambda_{\text {current }}^{0-}$, where $\lambda^{-0}\left(\lambda^{0-}\right)$ is the rate from $\mathrm{NV}^{-}$to $\mathrm{NV}^{0}$ $\left(\mathrm{NV}^{0}\right.$ to $\left.\mathrm{NV}^{-}\right)$. Furthermore, $\lambda_{\text {current }}^{-0}\left(\lambda_{\text {current }}^{0-}\right)$ and $\lambda_{\text {laser }}^{-0}\left(\lambda_{\text {laser }}^{0-}\right)$ are the rates originating from the current and the laser, respectively. In the photon accumulation measurements, the change in the equilibrium state from higher to lower photon counts can be fitted with a single exponential $e^{-\lambda t}$, as shown in the inset of Fig. 2(c). Thus, we can obtain the total rate $\left(\lambda=\lambda^{-0}+\lambda^{0-}\right)$. To independently estimate the rates from the laser and current, further experiments and analyses were performed, as described below.

First, we focused on the transition from $\mathrm{NV}^{-}$to $\mathrm{NV}^{0}$ during the current pulse in the measurement shown in the inset of Fig. 2(c). We estimated the dependence of $\lambda^{-0}$ on the laser power with a constant injected current. To solve the rate equation, we obtained the rate of $\lambda^{-0}$ as follows [Eq. (A11) in the Appendix]:

$$
\lambda^{-0}=p_{\mathrm{eqNV}^{0}} \cdot\left(\lambda^{-0}+\lambda^{0-}\right)=p_{\mathrm{eqNV}^{0}} \cdot \lambda .
$$

Here, $p_{\text {eqNV }}{ }^{0}$ is the equilibrium population of $\mathrm{NV}^{0}$ at the lower photon count in Fig. 2(c). The equilibrium population $p_{\text {eqNV }}{ }^{0}$ depends on the laser power and current. The dependence is observed as the depth of the dip in the experiment shown in Fig. 2(c) (see the Appendix). Therefore, $p_{\mathrm{eqNV}^{0}}$ was estimated from the following relationship with the depth of the dip:

$$
p_{\text {eqNV }}=0.568+\frac{0.373}{0.615} \cdot \text { Depth of the dip. }
$$

The details of the derivation are described in the Appendix. From $p_{\text {eqNV }}$, estimated with Eq. (2), the total rate $\lambda=\lambda^{-0}+\lambda^{0-}$ from the fitting of the results in Fig. 2(c) (inset) with a single exponential $e^{-\lambda t}$, and from Eq. (1), we obtained the rate $\lambda^{-0}=\lambda_{\text {current }}^{-0}+\lambda_{\text {laser }}^{-0}$. The dependence of $\lambda^{-0}$ on laser power with constant injection current is plotted in Fig. 3(a). The rate increases as the power of the 532-nm laser increases; moreover, when we fix the laser power and vary the current, the rate also increases. Thus, to estimate $\lambda_{\text {current }}^{-0}$, we fit the data points in Fig. 3(a) with the linear function $y=a+b x$, where $y$ and $x$ correspond to $\lambda^{-0}$ and the laser power, respectively, and the constant $a$ corresponds to $\lambda_{\text {current }}^{-0}$. The current dependence of $\lambda_{\text {current }}^{-0}$ is shown in Fig. 3(b). An increase of $\lambda_{\text {current }}^{-0}$ with current is observed, and the maximum rate is $\lambda_{\text {current }}^{-0}=0.72 \pm 0.10\left(\mu s^{-1}\right)$.

We measure $\lambda_{\text {current }}^{-0}+\lambda_{\text {laser }}^{-0}$ on another single NV center that resides $1 \mu \mathrm{m}$ away from the edge of the $n$-type layer. The measured values are in good agreement with the results
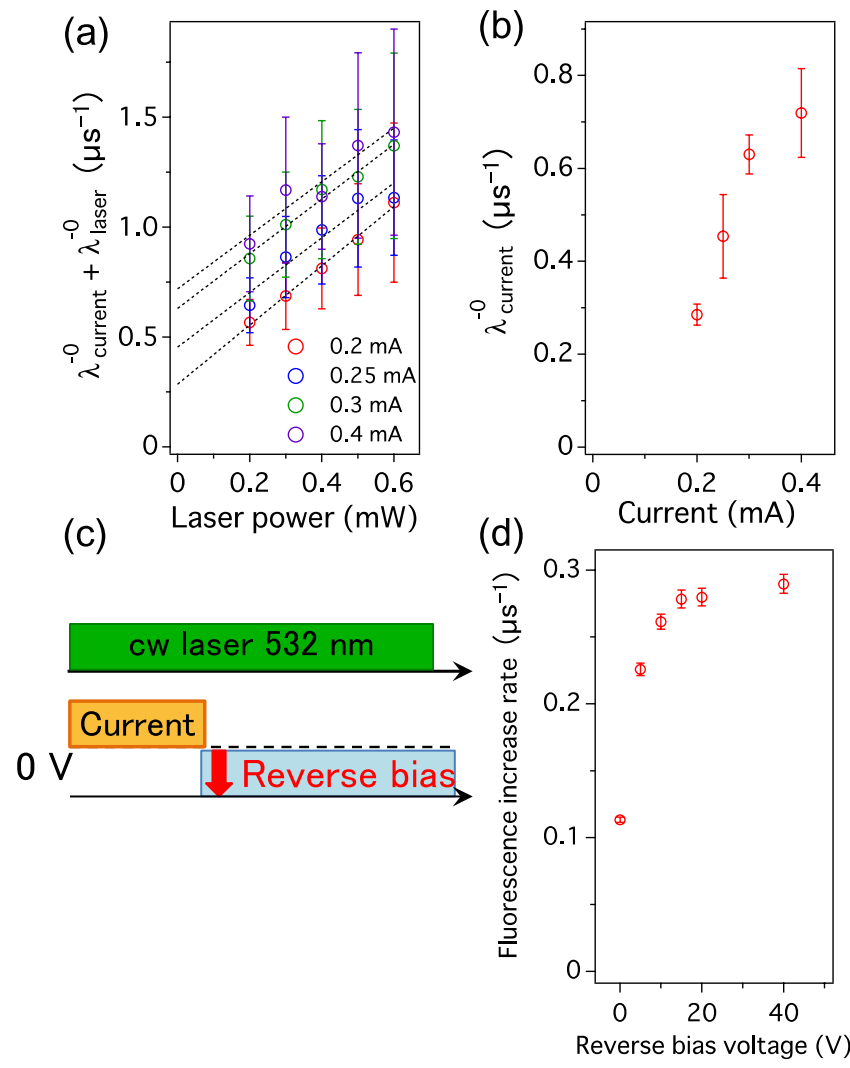

FIG. 3. (a) Dependence of the rate $\lambda_{\text {current }}^{-0}+\lambda_{\text {laser }}^{-0}$ on the laser power estimated from the experiment in Fig. 2(c). The dotted lines are the linear fitted lines for each injected current value. (b) Dependence of $\lambda_{\text {current }}^{-0}$ on the injected current. The values of $\lambda_{\text {current }}^{-0}$ were derived from those when the laser power is zero in the fitting of Fig. 3(a). (c) A pulse sequence to apply reverse bias voltage after forward bias. (d) Dependence of the fluorescence increase rate on the reverse bias voltage. A laser power was fixed to $0.4 \mathrm{~mW}$ during forward and reverse current injection. A forward bias current was fixed to $0.5 \mathrm{~mA}$.

shown in Fig. 3(a). The maximum discrepancy of the two measurements $\left(0.31 \mu \mathrm{s}^{-1}\right.$ at $\left.I=0.3 \mathrm{~mA}, P=0.3 \mathrm{~mW}\right)$ is smaller than the margins of error in Fig. 3(a).

The fluorescence increase immediately after the release of the forward bias in Fig. 2(c) is slower compared to the rate of the initial fluorescence decrease. In a $p$ - $n$ junction, the transient behavior is well known to depend on the storage of the minority carrier, where the diffusion time for the remaining minority carriers immediately after the release of the forward bias is affected [36]. In addition, the trapping of carriers by trapping centers in the bulk or the surface may also cause a slower change in fluorescence. If the minority carriers or trapped carriers are involved, the rate can be recovered by applying a reverse bias voltage. Hence, to investigate this point, we apply the reverse bias after the current pulse of the forward bias, as shown in Fig. 3(c), and the result obtained is shown in Fig. 3(d). In this experiment, we irradiated the NV center with a 532-nm laser of $0.4 \mathrm{~mW}$ during forward and reverse current 
injection, and the forward bias current was $0.5 \mathrm{~mA}$. Without reverse bias, the fluorescence increase rate $\left(\mathrm{NV}^{0}\right.$ to $\mathrm{NV}^{-}$) was $0.11 \mu \mathrm{s}^{-1}$; moreover, we also observed an increase in the rate with reverse bias. The saturated maximum rate was $0.29 \mu \mathrm{s}^{-1}$, which is close to the rate of the optical change at $0.4 \mathrm{~mW}$ (see the Appendix). This result supports the interpretation that the slower change in fluorescence is due to the storage of the minority carriers or trapped carriers. The fast recovery in the $p-i-n$ device by optical irradiation and the application of reverse bias voltage is important when considering the fast control of the charge states. Note that the reverse bias does not change the charge from $\mathrm{NV}^{0}$ to $\mathrm{NV}^{-}$in the dark after injecting the current.

\section{Nondestructive single-shot charge-state detection of individual $\mathrm{NV}$ centers}

We demonstrate that current injection not only changes the dynamic steady state but also the NV center charge state in the dark. Therefore, we quantitatively estimate the population of $\mathrm{NV}^{-}$via single-shot charge-state detection $[29,35,37]$. In this measurement, the low-power 593-nm laser illumination (about $1 \mu \mathrm{W}$ ) allows the direct and nondestructive observation of the NV charge state. The fluorescence time trace of single NV under such continuous illumination is shown in Fig. 4(a). Abrupt increases between high and low count rates $\left(\mathrm{NV}^{-}\right.$and $\mathrm{NV}^{0}$, respectively) are observed. By appropriately choosing the power and duration of the single laser pulse $(593 \mathrm{~nm})$, the number of fluorescence photons in this pulse allows the single-shot determination of the charge state. The histogram of the measurements shows two Poisson distributions corresponding to the fluorescence of $\mathrm{NV}^{0}$ and $\mathrm{NV}^{-}$. From the fitting of these two distributions with variable weights or areas, the ratio of this area yields the $\mathrm{NV}^{-}$and $\mathrm{NV}^{0}$ population. We repeated the single-shot charge-state measurements 2,000 times to obtain each histogram shown in Fig. 4.

We analyzed the action of a green laser pulse $(532 \mathrm{~nm})$, which is long enough to attain the dynamic steady state. After the laser is switched off, the charge state is measured using the 593-nm laser pulse. We obtained a population ratio of $p_{\text {eqNV-}}: p_{\text {eqNV0 }}=0.514: 0.486$, as shown in Fig. 4(b). This ratio is slightly lower than the reported value $\left(p_{\text {eqNV_- }}: p_{\text {eqNVo }}=0.75: 0.25\right)$ for a green laser $(532 \mathrm{~nm})$ [29]. We attribute the lower ratio to the carriers in the highly boron-doped $p$-type diamond in our samples. We confirmed that the ratio of $p_{\text {eqNV- }}: p_{\text {eqNVo }}$ is $0.735: 0.265$ in intrinsic diamond samples without a $p$-type diamond substrate (see the Appendix).

We calculated the readout fidelity from the area of the photon-count histogram, except for the overlap of the two Poisson distributions in Fig. 4(b). The calculated area is $92.7 \% \pm 0.93 \%$. The readout fidelity can be used to determine the charge state of the NV after one single-shot (a)

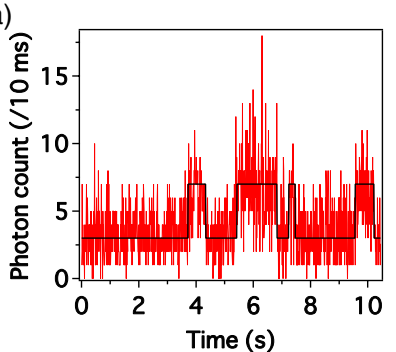

(b)

(c)

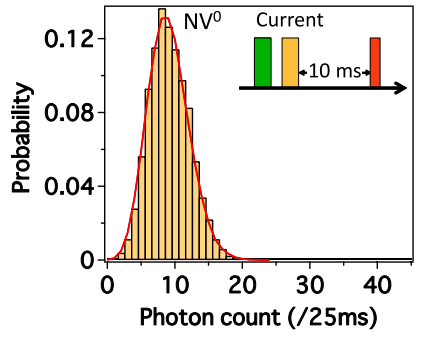

(d)

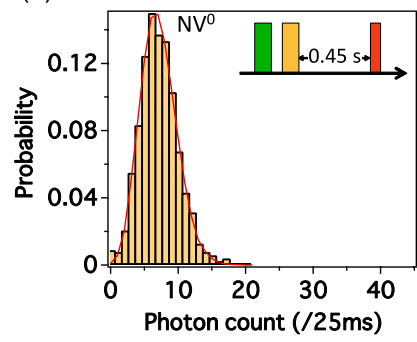

FIG. 4. (a) Time trace of the fluorescence of a single NV under continuous illumination with $593-\mathrm{nm}$ and $1-\mu \mathrm{W}$ laser light, showing the abrupt jumps between the two distinct states of $\mathrm{NV}$ : one with high $\left(\mathrm{NV}^{-}\right)$and one with low count rates $\left(\mathrm{NV}^{0}\right)$. (b) Histogram of photon counts during the 593-nm measurement pulse ( $1 \mu \mathrm{W}$ for $25 \mathrm{~ms})$ after illuminating the NV with a 532-nm and $100-\mu \mathrm{W}$ laser light for $20 \mathrm{~ms}$. Two solid black curves are obtained by curve fitting the Poisson distribution. The red solid curve is their sum. The blue line is the threshold that can be used to determine the charge state with one single-shot charge-state measurement. (c) Histogram of photon counts for the 593-nm measurement pulse, after illuminating the NV with a 532-nm and $0.1-\mathrm{mA}$ current injection. The waiting time between the injected current and detection laser pulse $(593 \mathrm{~nm})$ is set at $10 \mathrm{~ms}$. (d) Histogram of photon counts with the same pulse sequence as (c), but the waiting time is set at $0.45 \mathrm{~s}$. In Figs. 4(b)-4(d), we repeated the single-shot charge-state measurements 2,000 times to compile the histograms.

measurement to set the readout threshold at the intersection point of the two Poisson distributions.

\section{Deterministic purely electrical charge-state switching of individual NV centers}

We investigated the NV charge state after application of the bare current injection (i.e., in the dark without simultaneous 532-nm illumination). The Poisson distribution peak of $\mathrm{NV}^{-}$completely disappeared after the $0.1-\mathrm{mA}$ current [Fig. 4(c)], which shows the deterministic electrical chargestate control in the dark. We obtained the same results at the current range of $0.1-0.4 \mathrm{~mA}$. We repeated the current injections and single-shot charge-state measurements 2,000 times. Figures 4(c) and 4(d) show 100\% fidelity for the charge-state initialization.

Finally, the stability of $\mathrm{NV}^{0}$ in the dark and after the release of the bias voltage is investigated. The waiting time between the injected current and the detection laser pulse $(593 \mathrm{~nm})$ is increased. The histogram of the charge-state 
population remains unchanged even after $0.45 \mathrm{~s}$, as shown in Fig. 4(d); thus, $\mathrm{NV}^{0}$ is stable for more than $0.45 \mathrm{~s}$. Note that the measurement time is limited to $0.45 \mathrm{~s}$ by the equipment and not by the NV center.

The signatures tentatively attributed to $\mathrm{NV}^{+}$(lack of fluorescence) [26] were not observed in our sample. The lack of fluorescence was observed in the hydrogenated surface shallow $p$-type region. Thus, it is considered that the Fermi level at the NV center in our sample is not low enough to change to $\mathrm{NV}^{+}$.

\section{DISCUSSION}

Our measurements demonstrate that the single NV center maintains the $\mathrm{NV}^{0}$ charge state for more than $0.45 \mathrm{~s}$ in the dark after current injection. To estimate the maximum storage time, we compare the NV center with a P1 center, which is the nitrogen donor in the substitution position in the diamond lattice. The positively charged state after the optical change from the neutral state was sustained for longer than $12 \mathrm{~h} \mathrm{[38].} \mathrm{The} \mathrm{ionization} \mathrm{energy} \mathrm{level} \mathrm{of} \mathrm{the} \mathrm{NV}$ center from the valence band is estimated at around $3.0 \mathrm{eV}$ $[29,39,40]$ and is much larger than the donor level of the P1 center $(1.7 \pm 0.4 \mathrm{eV})[40]$. The $\mathrm{NV}^{0}$ charge state is possibly more stable than the $\mathrm{P} 1$ center and, in principle, can maintain its charge state longer than $12 \mathrm{~h}$ in the dark, which is interesting for single-electron memory [41]. For the charging time, a single hole carrier could be charged into a $\mathrm{NV}^{-}$ center within $1.39 \pm 0.20 \mu \mathrm{s}$, which is faster compared to previous studies on single-charge memory [41].

In our experiments, we obtained the maximum rate for electrical control from $\mathrm{NV}^{-}$to $\mathrm{NV}^{0}$ of $\lambda_{\text {current }}^{-0}=0.72 \pm$ $0.10\left(\mu s^{-1}\right)$. The recovery rate to the equilibrium charge state of $\mathrm{NV}^{-}$by optical control and the application of reverse bias voltage was estimated at $0.29 \mu \mathrm{s}^{-1}$. These values are important for creating quantum memories with lifetimes of seconds at room temperature [6]. Therefore, electron and nuclear spins need to be decoupled, which can be achieved by switching the hyperfine interaction $\left(\mathrm{A}_{/ /}=2.66 \mathrm{kHz}\right)$ with a much faster rate than the interaction itself (e.g., by charge-state switching [6]). Our rates are more than 250 and 100 times higher than the hyperfine interaction; therefore, they might allow for purely electrical decoupling without the detrimental laser heating of the sample.

In the $p-i-n$ diamond diode used in this study, the hole is the majority carrier. By introducing the highly phosphorous-doped $n$-type layer, electrons can be injected as the majority carrier and an electrically induced change from $\mathrm{NV}^{0}$ to $\mathrm{NV}^{-}$can be expected. In diamond Schottky$p n$ diodes, a switching time of nanoseconds has already been realized [34]. This switching time is much faster than the optical control rate, which is estimated to be $1.5 \mathrm{MHz}$ at $10 \mathrm{~mW}$. The improvement on the rates by electrical control to the tens of $\mathrm{MHz} \sim \mathrm{GHz}$ level may be achieved by using Schottky-diode structures. They can also be used for distant nuclear spins and close nuclear spins, and can potentially make $T_{2}$ longer than $1 \mathrm{~h}$.

\section{CONCLUSION}

We report the electrical control of a single $\mathrm{NV}^{-}$center by using a diamond diode for the first time. We manipulated the single $\mathrm{NV}^{-}$charge state on a solid-state device and investigated the charge-state dynamics of a single NV center by a nondestructive single-shot readout of the charge-state and time-resolved measurements. The fast and deterministic electrical control from the $\mathrm{NV}^{-}$to $\mathrm{NV}^{0}$ was demonstrated. Furthermore, we showed that the $\mathrm{NV}^{0}$ state in the dark is very stable after the release of the bias voltage, and the recovery to $\mathrm{NV}^{-}$by optical control was enhanced by applying reverse bias voltage. The electrical manipulation of the charge state between single $\mathrm{NV}^{-}$and $\mathrm{NV}^{0}$ will provide an important tool for using a single NV center for diamond-based spintronics and sensing applications by combining the spin properties of NV centers and the electrical control of qubits. Furthermore, by introducing the highly phosphorous-doped $n$-type layer, the electrons can be injected as majority carriers, and the electrically induced change from $\mathrm{NV}^{0}$ to $\mathrm{NV}^{-}$can be expected. These advances open the way for spins, qubits, and photon interface using fully controlled single atomic defects on devices at room temperature.

\section{ACKNOWLEDGMENTS}

The authors acknowledge financial support from the Japan Science and Technology KAKENHI (Grants No. 24102705 and No. 23681017), as well as the National Institute of Information and Communications Technology (NICT) program and SCOPE, the JST Core Research for Evolutional Science and Technology (CREST) program, the European Union (EU) via the Solid State Quantum Technology and Metrology Using Spins (SQUTEC) grants, DIAMANT and solid state systems for quantum information processing (SOLID), the Deutsche Forschungsgemeinschaft (DFG) via the research groups 730, 1482 and 1495, and the Max Planck Society. We thank N. Aslam, M. Pfender, and G. Waldherr for fruitful discussion.

\section{APPENDIX}

\section{Rate equations}

The charge-state dynamics of $\mathrm{NV}^{-}$and $\mathrm{NV}^{0}$ can be described by the following rate equations:

$$
\left(\begin{array}{c}
\dot{p}_{\mathrm{NV}^{-}} \\
\dot{p}_{\mathrm{NV}^{0}}
\end{array}\right)=\left(\begin{array}{cc}
-\lambda^{-0} & \lambda^{0-} \\
\lambda^{-0} & -\lambda^{0-}
\end{array}\right) \cdot\left(\begin{array}{c}
p_{\mathrm{NV}^{-}} \\
p_{\mathrm{NV}^{0}}
\end{array}\right)=K\left(\begin{array}{c}
p_{\mathrm{NV}^{-}} \\
p_{\mathrm{NV}^{0}}
\end{array}\right) .
$$

Here, $p_{\mathrm{NV}^{-}}$and $p_{\mathrm{NV}^{0}}$ are the populations of $\mathrm{NV}^{-}$and $\mathrm{NV}^{0}$, respectively, where $\lambda^{-0}$ and $\lambda^{0-}$ are the charge-state 
transition rates from $\mathrm{NV}^{-}$to $\mathrm{NV}^{0}$ and from $\mathrm{NV}^{0}$ to $\mathrm{NV}^{-}$, respectively. $K$ represents the rate matrix.

At the charge-state equilibrium, $\dot{p}_{\mathrm{NV}^{-}}=\dot{p}_{\mathrm{NV}^{0}}=0$ is satisfied. Hence, the following relation from Eq. (A1) can be derived:

$$
\lambda^{-0} p_{\mathrm{eqNV}}=\lambda^{0-} p_{\mathrm{eqNV}} .
$$

Here, $p_{\mathrm{eqNV}}{ }^{-}$and $p_{\mathrm{eqNV}}{ }^{0}$ are the equilibrium populations of $\mathrm{NV}^{-}$and $\mathrm{NV}^{0}$, respectively. In this case, the following relationship must be satisfied:

$$
p_{\mathrm{eqNV}^{-}}+p_{\mathrm{eqNV}}=1 .
$$

Using these terms, the relations between equilibrium populations and rates are given as

$$
\begin{aligned}
& p_{\mathrm{eqNV}^{-}}=\frac{\lambda^{0-}}{\lambda^{-0}+\lambda^{0-}} . \\
& p_{\mathrm{eqNV}^{0}}=\frac{\lambda^{-0}}{\lambda^{-0}+\lambda^{0-}} .
\end{aligned}
$$

Note that these equilibrium populations do not depend on the initial condition.

To solve the rate equation (A1), the solution can be written as

$$
\begin{aligned}
& p_{\mathrm{NV}^{-}}(t)=a_{1} \exp \left(\lambda_{1} t\right)+a_{2} \exp \left(\lambda_{2} t\right) \\
& p_{\mathrm{NV}^{0}}(t)=b_{1} \exp \left(\lambda_{1} t\right)+b_{2} \exp \left(\lambda_{2} t\right) .
\end{aligned}
$$

Here, the eigenvalues of $K$ are given by $\lambda_{1}=0$ and $\lambda_{2}=-\left(\lambda^{-0}+\lambda^{0-}\right)$. Therefore, both $p_{\mathrm{NV}^{-}}(t)$ and $p_{\mathrm{NV}^{0}}(t)$ can be written as

$$
\begin{aligned}
& p_{\mathrm{NV}^{-}}(t)=a_{1}+a_{2} \exp \left[-\left(\lambda^{-0}+\lambda^{0-}\right) t\right] \\
& p_{\mathrm{NV}^{0}}(t)=b_{1}+b_{2} \exp \left[-\left(\lambda^{-0}+\lambda^{0-}\right) t\right] .
\end{aligned}
$$

Here, $a_{1}$ and $b_{1}$ are equal to the equilibrium populations $p_{\text {eqNV }}{ }^{-}$and $p_{\text {eqNV }}$. Importantly, $a_{2}$ and $b_{2}$ are obtained from the initial conditions. Therefore, using an initial conditions $p_{\mathrm{NV}^{-}}(0)=1, \quad p_{\mathrm{NV}^{0}}(0)=0$, these constants would be

$$
\begin{gathered}
a_{1}+a_{2}=1 \quad \text { or } \quad a_{2}=1-\frac{\lambda^{0-}}{\lambda^{-0}+\lambda^{0-}}=\frac{\lambda^{-0}}{\lambda^{-0}+\lambda^{0-}} \\
b_{2}=-b_{1}=-\frac{\lambda^{-0}}{\lambda^{-0}+\lambda^{0-}} .
\end{gathered}
$$

From this, we can obtain the solution of the rate equations:

$$
\begin{aligned}
& p_{\mathrm{NV}^{-}}(t)=\frac{\lambda^{0-}}{\lambda^{-0}+\lambda^{0-}}+\frac{\lambda^{-0}}{\lambda^{-0}+\lambda^{0-}} \exp \left[-\left(\lambda^{-0}+\lambda^{0-}\right) t\right] \\
& p_{\mathrm{NV}^{0}}(t)=\frac{\lambda^{-0}}{\lambda^{-0}+\lambda^{0-}}-\frac{\lambda^{-0}}{\lambda^{-0}+\lambda^{0-}} \exp \left[-\left(\lambda^{-0}+\lambda^{0-}\right) t\right] .
\end{aligned}
$$

Using Eq. (A5), we can obtain $\lambda^{-0}$ by

$$
\lambda^{-0}=p_{\mathrm{eqNV}^{0}} \cdot\left(\lambda^{-0}+\lambda^{0-}\right)=p_{\mathrm{eqNV}^{0}} \cdot \lambda .
$$

Here, the total rate $\lambda$ can be attained to fit the experimental results using Eq. (A10). The equilibrium population of $\mathrm{NV}^{0}\left(p_{\text {eqNV }}\right)$ can be measured by the difference of fluorescence intensity between $\mathrm{NV}^{-}$and $\mathrm{NV}^{0}$.

\section{Synthesis of $p-i-n$ diamond}

Intrinsic ( $i$ ) and phosphorous ( $\mathrm{P})$-doped $n$-type layers were independently grown by microwave-plasmaenhanced chemical vapor deposition using a mixture of $\mathrm{CH}_{4}$ and $\mathrm{H}_{2}$ gas on high-pressure and high-temperature synthetic IIb-type (001) single-crystalline diamond with a misorientation angle of approximately $1^{\circ}$. The input microwave power and $\mathrm{CH}_{4} / \mathrm{H}_{2}$ ratio in $i$ - and $n$-type films were set to $4200 \mathrm{~W}$ and $750 \mathrm{~W}$ and $4.0 \%$ and $0.4 \%$, respectively. The gas pressures were 170 torr and 25 torr for the $i$ - and $n$-type films, respectively. The substrate temperatures were maintained constant at $850{ }^{\circ} \mathrm{C}$ and $900{ }^{\circ} \mathrm{C}$ for $i$ - and $n$-type films, respectively. In the IIb-type substrate, boron (B) was intentionally doped at a concentration of around $10^{19} \mathrm{~cm}^{-3}$. The Hall mobility was measured to be around $10 \mathrm{~cm}^{2} / \mathrm{V} \cdot \mathrm{s}$. For the $i$-layer, $\mathrm{O}_{2}$ was added in the feed gas with an $\mathrm{O}_{2} / \mathrm{CH}_{4}$ ratio of $2.5 \%$ to reduce the contamination by $\mathrm{B}$ and other impurity atoms. In the $i$-layer, the concentration of color centers was reduced to below $0.1 \mathrm{ppb}$ $\left(1 \times 10^{13} \mathrm{~cm}^{-3}\right)$. For the $n$-type layer, $\mathrm{PH}_{3}$ diluted with $\mathrm{H}_{2}$ was used for $\mathrm{P}$ doping. The typical doping concentration of $\mathrm{P}$ was around $10^{18} \mathrm{~cm}^{-3}$, with a $\mathrm{PH}_{3} / \mathrm{CH}_{4}$ ratio of $5 \%$ and a Hall mobility of around $150 \mathrm{~cm}^{2} / \mathrm{V} \cdot \mathrm{s}$. The thicknesses of the IIb substrate, $i$-layer, and $n$-type layer were $0.5 \mathrm{~mm}$, $10 \mu \mathrm{m}$, and $0.5 \mu \mathrm{m}$, respectively.

After growth, mesa structures with diameters of $220 \mu \mathrm{m}$ were fabricated by conventional photolithography and inductively coupled plasma (ICP) etching. Nitrogen (N) was subsequently implanted because the $i$-layer without such implantation is too pure to detect native NV centers (concentration of the NV centers is reduced to $1 \times 10^{7} \mathrm{~cm}^{-3}$ ). Hence, $\mathrm{N}$ was ion implanted at a depth of $300 \mathrm{~nm}$, with a density of $1 \times 10^{9}$ atom $/ \mathrm{cm}^{2}$, and a kinetic energy of $180 \mathrm{keV}$. Subsequent annealing at $800^{\circ} \mathrm{C}$ for $1 \mathrm{~h}$ produced the desired NV centers. The $p-i-n$ junction layers were kept in a mixture of $\mathrm{H}_{2} \mathrm{SO}_{4}$ and $\mathrm{HNO}_{3}$ at $200{ }^{\circ} \mathrm{C}$ for $60 \mathrm{~min}$ to remove any residual surface contamination and terminate surface oxygen atoms. Following this, $\operatorname{Ti}(30 \mathrm{~nm}) / \operatorname{Pt}(100 \mathrm{~nm}) / \mathrm{Au}(200 \mathrm{~nm})$ electrodes with diameters of $200 \mu \mathrm{m}$ or $1.5 \mathrm{~mm} \times 1.5 \mathrm{~mm}$ were formed on the $n$-type layer and IIb-type substrate, respectively. The electrodes on the $n$-type layer were connected with $\mathrm{Au}$ wires, and the $p$-type film electrode was attached to a $\mathrm{Cu}$ plate. Surface hydrogenation was carried out by irradiation with $\mathrm{H}_{2}$ radicals for $10 \mathrm{~min}$ at $4 \mathrm{kPa}$ using a hot filament system. The flow of high-purity $\mathrm{H}_{2}$ (9 grade) gas was set to $400 \mathrm{sccm}$. The sample temperature was maintained below 
$550{ }^{\circ} \mathrm{C}$ during hydrogenation. Oxidation was carried out by ICP etching in the presence of oxygen gas. The sample was annealed at $420^{\circ} \mathrm{C}$ for $30 \mathrm{~min}$ in an $\mathrm{Ar}$ environment. Finally, all electrical properties were measured in vacuum at room temperature.

\section{Experimental setup}

A programmable pulse generator was used to generate current pulses. Gold wires were connected to both the top and bottom electrodes. Charge-state manipulation was carried out on single NV centers within the $i$-layer near the edge of a mesa structure. The $p-i-n$ diamond exhibited an ideal diode characteristic with a rectification ratio of about $1 \times 10^{19}$ at $\pm 30 \mathrm{~V}$. A homebuilt confocal microscope was used to observe the single NV centers. All experiments were conducted under ambient conditions at room temperature.

\section{Charge-state population of undoped sample}

We performed charge-state population measurements with a weak orange (593-nm) laser on an undoped diamond sample. The measurement sequence and histogram of the photon count are shown in Fig. 5. In this measurement, the populations of $\mathrm{NV}^{0}$ and $\mathrm{NV}^{-}$were $26.5 \%$ and $73.5 \%$, respectively. These values are in good agreement with those in a previous report [29].

\section{Measurement of charge-state dynamics}

When conducting real-time observations of the chargestate changes, we were able to obtain a sufficient number of photons (100 k count/s) during the charge-state manipulation. In contrast, to investigate the dynamics of chargestate transitions, it was necessary to perform shorter timescale measurements, where the length of the total pulse sequence was about $100 \mu \mathrm{s}$. However, we could obtain only 10 photons from a NV center for such a pulse sequence. To collect sufficient photons to derive transition

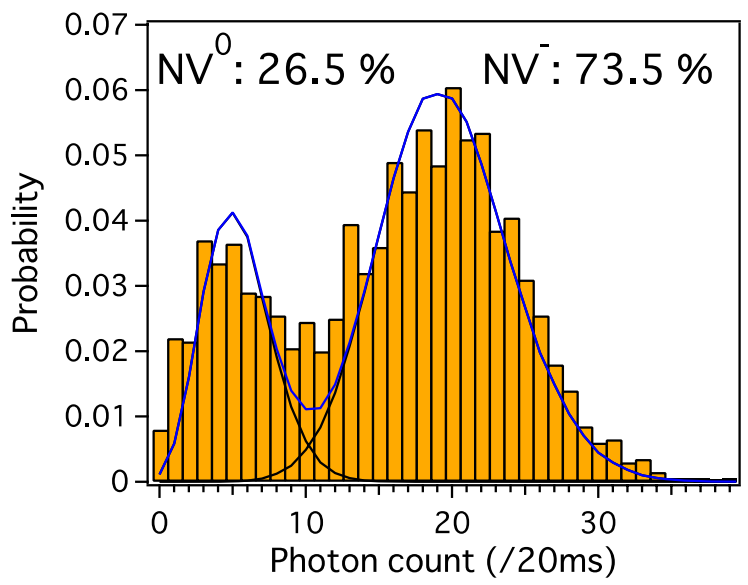

FIG. 5. Charge-state population of the undoped sample. rates and charge-state populations with high accuracy, we performed photon-accumulation measurements, which consisted of about $1 \times 10^{6}$ cycles of pulse sequences. In this measurement, a P7887 (FAST ComTec) counting board was employed, featuring a high-speed count rate of $4 \mathrm{GHz}$ and a time resolution of $250 \mathrm{ps}$.

\section{Estimation of $\boldsymbol{p}_{\text {eqNV }}{ }^{0}$}

It was not possible to directly measure $p_{\mathrm{eqNV}}{ }^{0}$ from the experimental data, as the laser $(\lambda=532 \mathrm{~nm})$ was continuously irradiated, preventing the omission of the laser effect on charge-state population. Therefore, we derived $p_{\text {eqNV }}{ }^{0}$ from the change of photon count as the charge-state population shifted. The measurements corresponding to this estimation are shown in Fig. 2(c), where the change of photon count is represented as the "depth of the dip."

Initially, we measured the relationship between the depth of the dip and charge-state population. A sequence of measurements and results are presented in Fig. 6, where the current $(0.4 \mathrm{~mA})$ and laser pulses $(0.3 \mathrm{~mW})$ were separated. Under our experimental conditions, we obtained a $61.5 \%$ increase in photon count as the maximum depth after irradiation. This corresponds to a charge-state change from $\mathrm{NV}^{0}$ to $\mathrm{NV}^{-}$. Moreover, from the charge-state population measurements [Figs. 4(b) and 4(c)], it was evident that the population of $\mathrm{NV}^{0}$ had increased from $48.6 \%$ to $100.0 \%$ (postcurrent injection). By combining the data collected from measurements in Fig. 2(c), the 61.5\% change of photon count corresponded to a $51.4 \%(100.0 \%-48.6 \%)$ shift of $\mathrm{NV}^{0}$ population. Thus, from the population detected [Fig. 2(c)], $p_{\text {eqNV }}{ }^{0}$ could be estimated as follows:

$$
p_{\mathrm{eqNV}^{0}}=0.486+\frac{0.514}{0.615} \cdot \text { Depth of the dip. }
$$

Note that the rise time of the laser pulse is limited by that of the acousto-optic modulator of $9 \mathrm{~ns}$, which is sufficiently rapid compared to the charge-state transition.

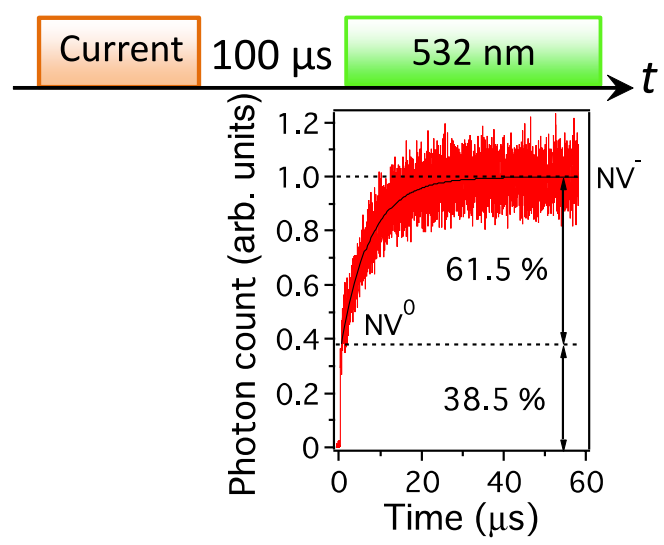

FIG. 6. Laser-induced charge-state transition from $\mathrm{NV}^{0}$ to $\mathrm{NV}^{-}$. 


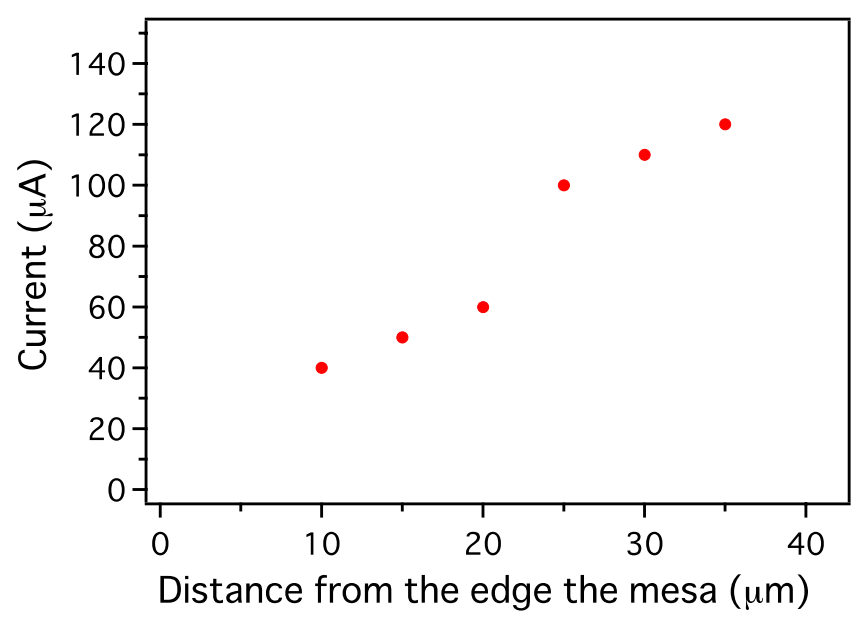

FIG. 7. Distance dependence of the charge-state change in current from $\mathrm{NV}^{-}$to $\mathrm{NV}^{0}$.

\section{Distance dependence of charge-state change}

Several single NV centers were measured, which were at different distances from the edge of a mesa structure. Depending on the distance examined, specific currents were required to alter the charge state. Figure 7 shows the distance dependence of a charge-state change from $\mathrm{NV}^{-}$to $\mathrm{NV}^{0}$ as a function of the measured current. Note that the experiment was performed near additional mesa, as discussed in the main text. In this test, we measured the currents that generated the same $p_{\text {eqNV }}$ for all NV centers at different distances. The results displayed an apparent distance dependence of the current, relating to the number of carriers (holes). In a previous study, we discussed carrier concentrations at the $\mathrm{NV}$ center in an $i$-layer [7]. In this case, the electric field decreased with a factor of approximately $\sin (\alpha)$, where $\alpha$ is the angle depth $(300 \mathrm{~nm}) /$ distance. For small $\alpha$, an almost linear dependence on the electric field (carrier concentration) can be expected. This rationale can be used to describe the approximately linear distance dependence of current change for the charge states.

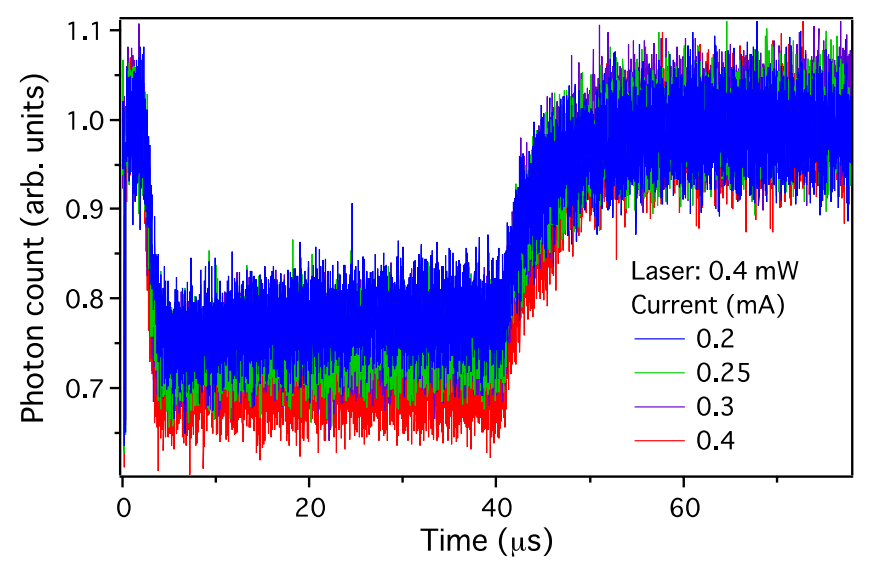

FIG. 8. Photon accumulation measurements of varying current values below the $0.4-\mathrm{mW}$ laser irradiation.

\section{Repetitive photon accumulation}

The repetitive photon accumulation data under varying currents are shown in Figs. 8 and 9. For these measurements, a current pulse of $40 \mu \mathrm{s}$ was applied under a fixed laser power of $0.4 \mathrm{~mW}$. In Fig. 8, we observe that the depth of the dip increased with current. This implies that the equilibrium population of $\mathrm{NV}^{0}$ increases with current.

Figure 9 shows the change in the total rate of equilibrium-state transition. The rate was determined as $1.18 \pm$ $0.27\left(\mu \mathrm{s}^{-1}\right)$ at $0.2 \mathrm{~mA}$ and increased to $1.54 \pm 0.32\left(\mu \mathrm{s}^{-1}\right)$ at $0.4 \mathrm{~mA}$. Meanwhile, the dip depth increased from $24 \%$ to $30.2 \%$.

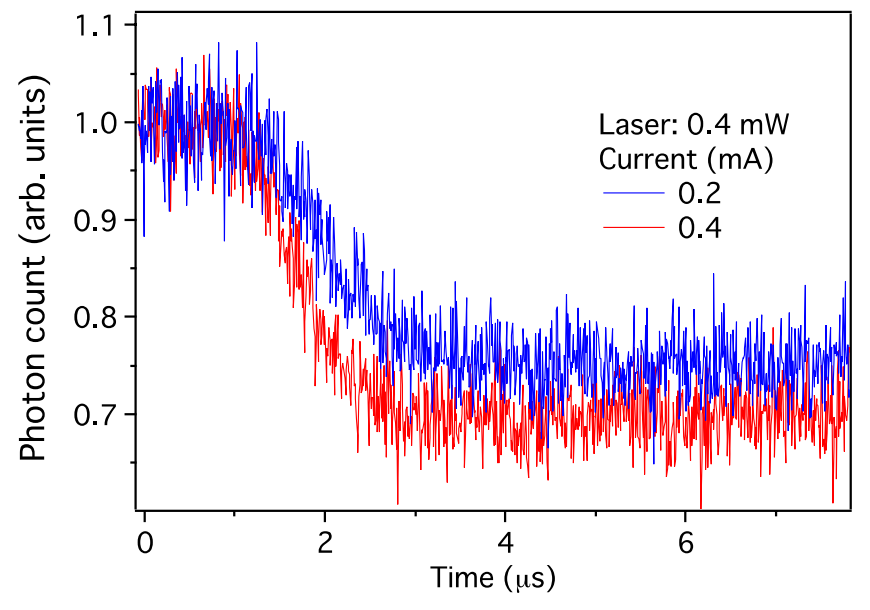

FIG. 9. Change in the total transition rate.

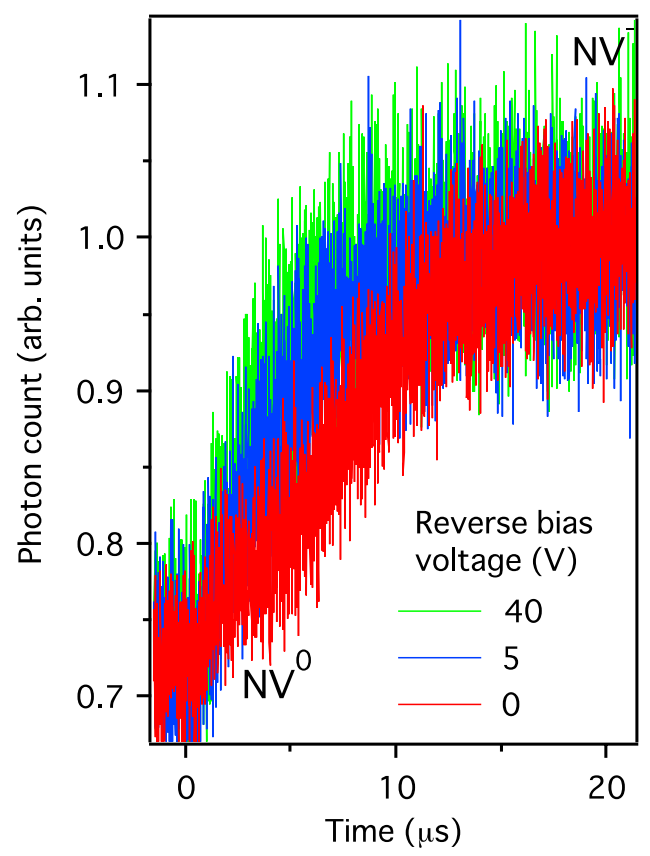

FIG. 10. Reverse bias voltage dependence on fluorescence increase rate. 


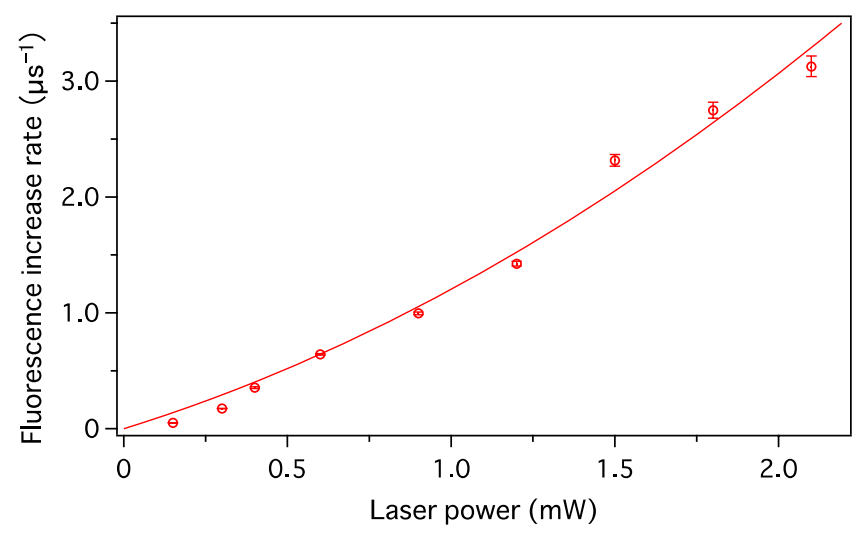

FIG. 11. Plot of laser power dependence of fluorescence increase rate.

\section{Reverse bias and fluorescence increase rate}

Figure 10 shows the dependence of fluorescence increase rate under the influence of a reverse bias voltage. The measurement was conducted using $0.4 \mathrm{~mW}$ laser illumination, and the corresponding pulse sequence is shown in Fig. 3.

When the reverse bias voltage was set to zero, the photon count recovered after the forward bias voltage was switched off (because of optical changes in the charge state), as shown in Fig. 6. Once the reverse bias voltage was applied, the fluorescence was observed to increase at a significantly rapid rate, depending on the reverse bias voltage. The resulting plot of voltage versus the fluorescence rate is shown in Fig. 3(d), and further details of the observed trend are discussed in the main text.

\section{Laser power dependence of fluorescence increase rate}

A fluorescence increase of a NV, displayed in Fig. 6 only contains optical-bidirectional rates. Laser power dependence of fluorescence increase rate (reverse of the time constant of fluorescence increase) is displayed in Fig. 11. The data are fitted by the function $f(I)=a I+b I^{2}$, affording the value of $a=0.893 \pm 0.189 /(\mu \mathrm{s} \cdot \mathrm{mW})$ and $b=0.319 \pm 0.109 /\left(\mu \mathrm{s} \cdot \mathrm{mW}^{2}\right)$.

[1] J. J. Pla, K. Y. Tan, J. P. Dehollain, W. H. Lim, J. J. L. Morton, D. N. Jamieson, A. S. Dzurak, and A. Morello, A Single-Atom Electron Spin Qubit in Silicon, Nature (London) 489, 541 (2012).

[2] A. Morello, J. J. Pla, F. A. Zwanenburg, K. W. Chan, K. Y. Tan, H. Huebl, M. Möttönen, C. D. Nugroho, C. Yang, J. A. van Donkelaar, A. D. C. Alves, D. N. Jamieson, C. C. Escott, L. C. L. Hollenberg, R. G. Clark, and A. S. Dzurak, Single-Shot Readout of an Electron Spin in Silicon, Nature (London) 467, 687 (2010).
[3] T. Hayashi, T. Fujisawa, H. D. Cheong, Y. H. Jeong, and Y. Hirayama, Coherent Manipulation of Electronic States in a Double Quantum Dot, Phys. Rev. Lett. 91, 226804 (2003).

[4] T. Fujisawa, T. Hayashi, R. Tomita, and Y. Hirayama, Bidirectional Counting of Single Electrons, Science 312, 1634 (2006).

[5] H. Bernien, B. Hensen, W. Pfaff, G. Koolstra, M. S. Blok, L. Robledo, T. H. Taminiau, M. Markham, D. J. Twitchen, L. Childress, and R. Hanson, Heralded Entanglement between Solid-State Qubits Separated by Three Metres, Nature (London) 497, 86 (2013).

[6] P. C. Maurer, G. Kucsko, C. Latta, L. Jiang, N. Y. Yao, S. D. Bennett, F. Pastawski, D. Hunger, N. Chisholm, M. Markham, D. J. Twitchen, J. I. Cirac, and M. D. Lukin, Room-Temperature Quantum Bit Memory Exceeding One Second, Science 336, 1283 (2012).

[7] N. Mizuochi, T. Makino, H. Kato, D. Takeuchi, M. Ogura, H. Okushi, M. Nothaft, P. Neumann, A. Gali, F. Jelezko, J. Wrachtrup, and S. Yamasaki, Electrically Driven SinglePhoton Source at Room Temperature in Diamond, Nat. Photonics 6, 299 (2012).

[8] T. M. Babinec, B. J. M. Hausmann, M. Khan, Y. Zhang, J. R. Maze, P. R. Hemmer, and M. Lončar, A Diamond Nanowire Single-Photon Source, Nat. Nanotechnol. 5, 195 (2010).

[9] P. Neumann, N. Mizuochi, F. Rempp, P. R. Hemmer, H. Watanabe, S. Yamasaki, V. Jacques, T. Gaebel, F. Jelezko, and J. Wrachtrup, Multipartite Entanglement Among Single Spins in Diamond, Science 320, 1326 (2008).

[10] J. R. Maze, P. L. Stanwix, J. S. Hodges, S. Hong, J. M. Taylor, P. Cappellaro, L. Jiang, M. V. G. Dutt, E. Togan, A. S. Zibrov, A. Yacoby, R. L. Walsworth, and M. D. Lukin, Nanoscale Magnetic Sensing with an Individual Electronic Spin in Diamond, Nature (London) 455, 644 (2008); G. Balasubramanian, I. Y. Chan, R. Kolesov, M. Al-Hmoud, J. Tisler, C. Shin, C. Kim, A. Wojcik, P. R. Hemmer, A. Krueger, T. Hanke, A. Leitenstorfer, R. Bratschitsch, F. Jelezko, and J. Wrachtrup, Nanoscale Imaging Magnetometry with Diamond Spins under Ambient Conditions, Nature (London) 455, 648 (2008).

[11] P. Maletinsky, S. Hong, M. S. Grinolds, B. Hausmann, M. D. Lukin, R. L. Walsworth, M. Lončar, and A. Yacoby, A Robust Scanning Diamond Sensor for Nanoscale Imaging with Single Nitrogen-Vacancy Centres, Nat. Nanotechnol. 7, 320 (2012).

[12] V. M. Acosta, E. Bauch, M. Ledbetter, C. Santori, K. M. Fu, P. Barclay, R. G. Beausoleil, H. Linget, J.-F. Roch, F. Treussart, S. Chemerisov, W. Gawlik, and D. Budker, Diamonds with a High Density of Nitrogen-Vacancy Centers for Magnetometry Applications, Phys. Rev. B 80, 115202 (2009).

[13] H. J. Mamin, M. Kim, M. H. Sherwood, C. T. Rettner, K. Ohno, D. D. Awschalom, and D. Rugar, Nanoscale Nuclear Magnetic Resonance with a Nitrogen-Vacancy Spin Sensor, Science 339, 557 (2013); T. Staudacher, F. Shi, S. Pezzagna, J. Meijer, J. Du, C. A. Meriles, F. Reinhard, and J. Wrachtrup, Nuclear Magnetic Resonance Spectroscopy on a (5-Nanometer $)^{3}$ Sample Volume, Science 339, 561 (2013).

[14] L. T. Hall, C. D. Hill, J. H. Cole, B. Stadler, F. Caruso, P. Mulvaney, J. Wrachtrup, and L. C. L. Hollenberg, Monitoring Ion-Channel Function in Real Time through Quantum 
Decoherence, Proc. Natl. Acad. Sci. U.S.A. 107, 18777 (2010).

[15] L. P. McGuinness, Y. Yan, A. Stacey, D. A. Simpson, L. T. Hall, D. Maclaurin, S. Prawer, P. Mulvaney, J. Wrachtrup, F. Caruso, R. E. Scholten, and L. C. L. Hollenberg, Quantum Measurement and Orientation Tracking of Fluorescent Nanodiamonds inside Living Cells, Nat. Nanotechnol. 6, 358 (2011).

[16] D. Le Sage, K. Arai, D. R. Glenn, S. J. DeVience, L. M. Pham, L. Rahn-Lee, M. D. Lukin, A. Yacoby, A. Komeili, and R. L. Walsworth, Optical Magnetic Imaging of Living Cells, Nature (London) 496, 486 (2013).

[17] N. Mizuochi, P. Neumann, F. Rempp, J. Beck, V. Jacques, P. Siyushev, K. Nakamura, D. J. Twitchen, H. Watanabe, and S. Yamasaki, Coherence of Single Spins Coupled to a Nuclear Spin Bath of Varying Density, Phys. Rev. B 80, 041201 (2009).

[18] A. Gruber, A. Dräbenstedt, C. Tietz, L. Fleury, J. Wrachtrup, and C. Von Borczyskowski, Scanning Confocal Optical Microscopy and Magnetic Resonance on Single Defect Centers, Science 276, 2012 (1997); R. J. Epstein, F. M. Mendoza, Y. K. Kato, and D. D. Awschalom, Anisotropic Interactions of a Single Spin and Dark-Spin Spectroscopy in Diamond, Nat. Phys. 1, 94 (2005).

[19] K. Iakoubovskii, G. J. Adriaenssens, and M. Nesladek, Photochromism of Vacancy-Related Centres in Diamond, J. Phys. Condens. Matter 12, 189 (2000).

[20] N. B. Manson and J. Harrison, Photo-ionization of the Nitrogen-Vacancy Center in Diamond, Diam. Relat. Mater. 14, 1705 (2005).

[21] T. Gaebel, M. Domhan, C. Wittmann, I. Popa, F. Jelezko, J. R. Rabeau, A. D. Greentree, S. Prawer, E. Trajkov, and P. Hemmer, Photochromism in Single Nitrogen-Vacancy Defect in Diamond, Appl. Phys. B: Lasers Opt. 82, 243 (2006).

[22] G. Waldherr, J. Beck, M. Steiner, P. Neumann, A. Gali, T. Frauenheim, F. Jelezko, and J. Wrachtrup, Dark States of Single Nitrogen-Vacancy Centers in Diamond Unraveled by Single Shot NMR, Phys. Rev. Lett. 106, 157601 (2011).

[23] K.-M. C. Fu, C. Santori, P. E. Barclay, and R. G. Beausoleil, Conversion of Neutral Nitrogen-Vacancy Centers to Negatively Charged Nitrogen-Vacancy Centers through Selective Oxidation, Appl. Phys. Lett. 96, 121907 (2010).

[24] C. Bradac, T. Gaebel, N. Naidoo, M. J. Sellars, J. Twamley, L. J. Brown, A. S. Barnard, T. Plakhotnik, A. V. Zvyagin, and J. R. Rabeau, Observation and Control of Blinking Nitrogen-Vacancy Centres in Discrete Nanodiamonds, Nat. Nanotechnol. 5, 345 (2010).

[25] M. V. Hauf, B. Grotz, B. Naydenov, M. Dankerl, S. Pezzagna, J. Meijer, F. Jelezko, J. Wrachtrup, M. Stutzmann, F. Reinhard, and J. A. Garrido, Chemical Control of the Charge State of Nitrogen-Vacancy Centers in Diamond, Phys. Rev. B 83, 081304 (2011).

[26] B. Grotz, M. V. Hauf, M. Dankerl, B. Naydenov, S. Pezzagna, J. Meijer, F. Jelezko, J. Wrachtrup, M. Stutzmann, F. Reinhard, and J. A. Garrido, Charge State Manipulation of Qubits in Diamond, Nat. Commun. 3, 729 (2012).

[27] H. Kato, M. Wolfer, C. Schreyvogel, M. Kunzer, W. MüllerSebert, H. Obloh, S. Yamasaki, and C. E. Nebel, Tunable Light Emission from Nitrogen-Vacancy Centers in Single
Crystal Diamond PIN Diodes, Appl. Phys. Lett. 102, 151101 (2013).

[28] A. Lohrmann, S. Pezzagna, I. Dobrinets, P. Spinicelli, V. Jacques, J.-F. Roch, J. Meijer, and A. M. Zaitsev, Diamond Based Light-Emitting Diode for Visible Single-Photon Emission at Room Temperature, Appl. Phys. Lett. 99, 251106 (2011).

[29] N. Aslam, G. Waldherr, P. Neumann, F. Jelezko, and J. Wrachtrup, Photo-Induced Ionization Dynamics of the Nitrogen Vacancy Defect in Diamond Investigated by Single-Shot Charge State Detection, New J. Phys. 15, 013064 (2013).

[30] V. M. Acosta, C. Santori, A. Faraon, Z. Huang, K.-M. C. Fu, A. Stacey, D. A. Simpson, K. Ganesan, S. TomljenovicHanic, A. D. Greentree, S. Prawer, and R. G. Beausoleil, Dynamic Stabilization of the Optical Resonances of Single Nitrogen-Vacancy Centers in Diamond, Phys. Rev. Lett. 108, 206401 (2012).

[31] P. Siyushev, H. Pinto, M. Voros, A. Gali, F. Jelezko, and J. Wrachtrup, Optically Controlled Switching of the Charge State of a Single Nitrogen-Vacancy Center in Diamond at Cryogenic Temperatures, Phys. Rev. Lett. 110, 167402 (2013).

[32] A. Stacey, D. A. Simpson, T. J. Karle, B. C. Gibson, V. M. Acosta, Z. Huang, K.-M. C. Fu, C. Santori, R. G. Beausoleil, L. P. McGuinness, K. Ganesan, S. Tomljenovic-Hanic, A. D. Greentree, and S. Prawer, Near-Surface Spectrally Stable Nitrogen Vacancy Centres Engineered in Single Crystal Diamond, Adv. Mater. 24, 3333 (2012).

[33] V. M. Acosta, E. Bauch, M. P. Ledbetter, A. Waxman, L. S. Bouchard, and D. Budker, Temperature Dependence of the Nitrogen-Vacancy Magnetic Resonance in Diamond, Phys. Rev. Lett. 104, 070801 (2010).

[34] T. Makino, S. Tanimoto, Y. Hayashi, H. Kato, N. Tokuda, M. Ogura, D. Takeuchi, K. Oyama, H. Ohashi, H. Okushi, and S. Yamasaki, Diamond Schottky-pn Diode with High Forward Current Density and Fast Switching Operation, Appl. Phys. Lett. 94, 262101 (2009).

[35] G. Waldherr, P. Neumann, S. Huelga, F. Jelezko, and J. Wrachtrup, Violation of a Temporal Bell Inequality for Single Spins in a Diamond Defect Center, Phys. Rev. Lett. 107, 090401 (2011).

[36] S. M. Sze and K. K. Ng, Physics of Semiconductor Devices (John Wiley \& Sons, New York, 2006).

[37] X.-D. Chen, C.-L. Zou, F.-W. Sun, and G.-C. Guo, Optical Manipulation of the Charge State of Nitrogen-Vacancy Center in Diamond, Appl. Phys. Lett. 103, 013112 (2013).

[38] C. F. O. Graeff, E. Rohrer, C. E. Nebel, M. Stutzmann, H. Güttler, and R. Zachai, Optical Excitation of Paramagnetic Nitrogen in Chemical Vapor Deposited Diamond, Appl. Phys. Lett. 69, 3215 (1996).

[39] J. W. Steeds, S. J. Charles, J. Davies, and I. Griffin, Photoluminescence Microscopy of TEM Irradiated Diamond, Diam. Relat. Mater. 9, 397 (2000).

[40] J. P. Goss, P. R. Briddon, R. Jones, and S. Sque, Donor and Acceptor States in Diamond, Diam. Relat. Mater. 13, 684 (2004).

[41] L. Guo, E. Leobandung, and S. Y. Chou, A Silicon SingleElectron Transistor Memory Operating at Room Temperature, Science 275, 649 (1997). 